\title{
THE SPECIFICITY OF EMOTIONAL LABOR OF SOCIAL WORKERS HELPING CHILDREN
}

\author{
Irina Tarkhanova \\ Yaroslavl State Pedagogical University named after K.D. Ushinsky, \\ Russian Federation \\ Olga Koryakovtseva \\ Yaroslavl State Pedagogical University named after K.D. Ushinsky, \\ Russian Federation \\ Lyudmila Zhedunova \\ Yaroslavl State Pedagogical University named after K.D. Ushinsky, \\ Russian Federation \\ Irena Upeniece \\ Rīga Stradiņš University, Latvia
}

\begin{abstract}
The article presents the results of the study of the emotional perception of clients conducted among Russian social workers who help children. The research is based on the concept of the role of emotional labor in professional activities. At the same time, we proceed from the understanding that the content and volume of emotional labor depends on the type of clients of social workers: its specificity when working with children will not be the same as when working with adults. The results of the research show that social workers who help children have difficulties in regulating emotions when interacting with clients, and therefore in assessing children's actions and behavior. The results of the study show that the effectiveness of emotional labor of social workers helping children depends on the level of knowledge of the age characteristics of children and the pedagogical mechanisms of communicating with them. We conclude that we should pay more attention to the role of emotional labor in everyday social work with children.
\end{abstract}

Keywords: emotional labor, emotional perception of the client, social work with children, social worker.

\section{Introduction}

The competent approach in personnel training, selection and evaluation of personnel, in contrast to other approaches, focuses on identifying certain characteristics (qualities) of the person that provide the ability to do something, successfully cope with individual tasks and activities in general (Gruzdev, Kasakova, Kuznetsova, \& Tarkhanova 2018). The professional activity of the social worker is a manifestation of helping behavior. Helping behavior belongs 
to the category of the prosocial activity and includes any actions related to voluntary assistance or the intention to help other people regardless of the nature of its motives. Social assistance to children due to the high demands, special responsibility and emotional loads, potentially contains the danger of heavy feelings associated with dissatisfaction with professional activities and the probability of professional stress. Refusal of expression in communication with children and the ability to perceive the children's behavior through the prism of social assistance are heavy emotional labor. The ability to understand one's own feelings and manage emotions is now considered by many researchers as professionally important qualities of a social worker. We proceed from the understanding that these qualities are subjects to development in the course of professional activity, and this process forms the basis of the emotional labor of the social worker.

The purpose of this article is to study the emotional perception of children's by social workers, and on the basis of the specificity of this perception, the essence and content of emotional labor of social workers who help children are to be determined.

The object of the research: the process of emotional labor of social workers who help children.

The subject of the research: emotional perception of children's as the basis of the content of emotional labor of social workers who help children.

\section{The theoretical positions}

Effective social assistance requires effective interaction between the social worker and the client. In order to achieve the goals of social assistance, the social worker consciously control emotions, using them as a means of social interaction. When working with the client it is necessary to stay calm, it is unallowable to lose temper, even if the client causes the social worker to feel negatively, the social worker must be friendly even in situations of reaction to the anger and aggression of the client. Thus, during the working day the social worker repeatedly performs actions aimed at changing the intensity or qualitative characteristics of his/her own emotions, that is, the social worker does emotional labor.

In the end of the $\mathrm{XX}^{\text {th }}$ century, the American sociologist A.R. Hochschild (1979) put forward the concept of the sociality of human emotions. Her research emphasizes that individuals know what emotions should be experienced/detected in a given situation. At the same time, the author claims that work on emotions is carried out within the emotional culture that is formed in society as a whole and in the group to which the individual belongs, in particular. 
A. Hochschild describes two norms of the emotional culture of society: "feeling rules and display rules" (Hochschild, 1979). The first norm prescribes what emotions, of what intensity and duration should be experienced in this situation, the second norm dictates when and how emotions should be expressed. A. Hochschild notes: "When individuals follow the display rules, they are involved in surface acting, for example, we often "put on a smile" when it is required to greet a friend. With deep acting, the individual makes efforts to really experience the expected emotions; the author calls such efforts emotional labor" (Hochschild, 1979).

Emotional labor is an integral part of the professional activity, that is, it is a part of paid work. Emotional labor is especially noticeable in those professions that are characterized by "face to face" interaction, the desire to influence the worker's emotional state on the client's emotional state. In many ways, it is about caring professions, which includes the work of social workers. Specialists of the social sphere are encouraged to depict and experience the "right" emotions with the aim of influencing the client.

Hochschild (2003) believes that amount and intensity of emotional labor is increasing in modern society. Workers in accordance with the requirements of professional ethics suppress their real emotions and depict or excite the necessary emotions in order to improve the effectiveness of interaction with the client. It should be understood that when the expressed feelings regularly differ from the internal feelings, emotional dissonance is very likely, which has negative consequences, including those expressed in the form of emotional burnout. Hochschild (2011) believes that dissonance is an identity problem and reflects in her works on ways to overcome it.

In the modern understanding, "emotional labor reflects the action of different rules of feeling and different motivations that are regulated in social situations and relationships that are inextricably linked to the wider social context: institutions, power and the stratification system” (Bolton, 2009, p. 544).

In recent years, the notion of emotional labor within the framework of professional relations has significantly enriched: new variables, causes and aspects of these phenomena have been identified, new classifications have appeared (Taylor \& Wingfield, 2010; Vandysheva, 2014; Kanasz \& Zeilinska, 2017). Scientists clarify that this concept should not be applied to all kinds of occupations and professions and that for each labor context a detailed analysis of the factors affecting emotional labor is needed. One of such factors is the emotional perception of the client, which is natural: if the client is initially perceived positively, then the intensity of emotional labor is lower, if the client's perception is negative, the specialist has to make a lot of efforts to regulate his/her own emotional background, that is, to carry out intense emotional labor. 


\section{Materials and methods}

A specific part of the emotional labor of the social worker who helps children is interaction with undisciplined children's. The intensity of emotional labor in this case depends on what the children are in the perception of specialists. The identification of the subjective image of the child who is in need of social assistance was carried out by us using the scales of the semantic differential.

The method of semantic differential (Osgood, 1976) is a modified procedure of subjective scaling. The respondent is asked to evaluate the object (children) with the help of the proposed set of pairs of adjectives. The respondent is asked to underline one word in each pair of adjectives, which, in his/her opinion, more characterizes the "object": active - passive, strong - weak, good - bad, etc. A set of adjectives is bipolar (the respondent chooses one of two possible evaluation poles).

The research was conducted in January-March 2018, 62 social workers working in the services to help children in a difficult life situation took part in it. Sex of respondents is: $93 \%$ of women, $7 \%$ of men; work experience in social services is from 3 to 18 years. In follow-up personal interviews, we asked teachers to identify problems of interaction with children that require the most intense emotional labor.

To process the results of the research, methods of mathematical statistics were used: primary statistics, single-factor analysis of variance, factor analysis and correlation analysis. Data processing of the empirical study was carried out using the STATISTICA 6.0, statistical software package.

\section{Results and discussion}

Figure 1 shows the average profile of emotional perception of children's by social workers who participated in the study.

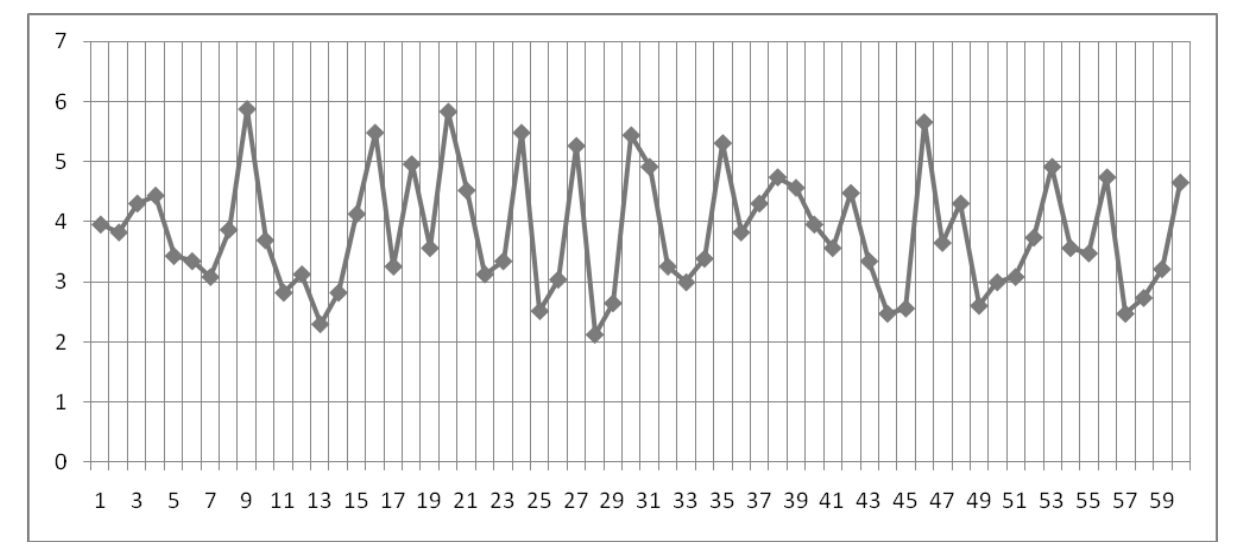

Figure 1 Profile of emotional perception of children's by social workers (X-axis-respondents, $Y$-axis-level of emotional perception of children) 
According to Figure 1, it can be concluded that the emotional perception of children's is mainly within the average values, which indicates a fairly calm attitude of most social workers to the traits of children. At the same time, on the profile it is possible to single out diagnostic points that indicate a more pronounced emotional reaction of specialists to certain traits of children's.

Thus, the following traits of children are perceived most emotionally: noisy, selfish, untidy, scatter brain, immoral, stubborn, indifferent, superficial, aggressive, anxious, rude, irresponsible. The rating of the emotional perception of these traits of children's is shown in Figure 2.

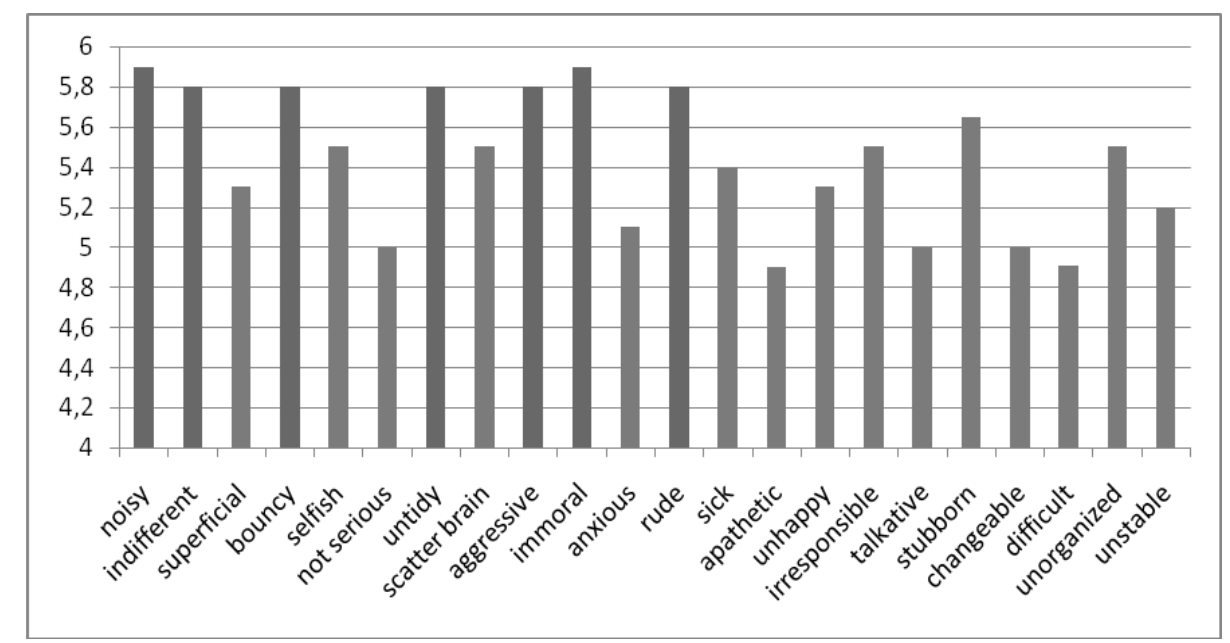

Figure 2 Rating of emotionality of perception of traits of children (X-axis - children's character traits, Y-axis-the level of emotional perception of children's character traits)

As can be seen in Figure 2, the greatest emotionality is the perception of such traits as: noisy, indifferent, bouncy, untidy, aggressive, immoral, rude.

This creates difficulties for specialists in managing the emotional side of interaction, and can also create difficulties in establishing an emotionally warm atmosphere. The main difficulty experienced by social workers in their emotional labor is the establishment of a calm and friendly communication with children who have the above traits.

At the same time, the indicator "insider - outsider" on the average shows the expression vector towards the "insider" characteristic, although it has one of the highest dispersion coefficients, that is, the majority of the respondents perceive the children's as "insider", but there are respondents, who noted high scores on the pole "outsider". At the same time, children's are perceived as sick, unhappy, etc., so specialists generally do not have emotional rejection of children, there is an empathic attitude towards children's. This gives grounds to believe that the correction of some negative moments of a socio-psychological 
nature in the relationship between social workers and their children's can be carried out with rather high efficiency.

The factor analysis was carried out to identify the factors that determine the perception of children by social workers.

The first factor $(17 \%)$ is represented by negative characteristics of the children's image and can be interpreted as "Failure to meet the requirements of the adult”; it is expressed primarily in such characteristics of the state of children in which strict discipline is impossible.

Table 1 The factor "Inability to meet the requirements of the adult"

\begin{tabular}{|l|c|}
\hline \multicolumn{1}{|c|}{ Statements } & Factor loads \\
\hline stereotyped-creative & 0,62 \\
\hline dependent-independent & 0,60 \\
\hline superficial -wise & 0,58 \\
\hline ordinary-unique & 0,56 \\
\hline disharmonious-harmonious & 0,50 \\
\hline serious-not serious & $-0,79$ \\
\hline cheerful-tired & 0,77 \\
\hline attentive-inattentive & $-0,76$ \\
\hline resolute-irresolute & $-0,71$ \\
\hline active-inactive & $-0,65$ \\
\hline happy-unhappy & $-0,65$ \\
\hline tidy-untidy & $-0,65$ \\
\hline caring- indifferent & $-0,62$ \\
\hline
\end{tabular}

If the social worker sees these traits in the children, he/she feels antipathy towards him/her and, accordingly, makes a lot of efforts to demonstrate friendly attitude. Such perception of children entails unbelief in the success of working with them; the specialist has a feeling of excessive difficulty in work, which means that dissatisfaction with work arises. At the same time, the negative perception of the children leads to mistakes in emotional labor and can lead to violations of pedagogical tact, bias in assessing the child's actions.

Table 2 Factor "Emotional safety of interaction with children's"

\begin{tabular}{|l|c|}
\hline \multicolumn{1}{|c|}{ Statements } & Factor loads \\
\hline hostile-friendly & 0,64 \\
\hline cruel - kind-hearted & 0,62 \\
\hline hard-responsive & 0,62 \\
\hline harmful-harmless & 0,55 \\
\hline dangerous-safe & 0,51 \\
\hline cheerful-sad & $-0,50$ \\
\hline loving-hating & $-0,58$ \\
\hline
\end{tabular}


The second important factor (15\%) is represented by such characteristics of the children's' image, which relate to the possibility of emotionally safe interaction; do not threaten the violation of the internal emotional stability of the social worker. It can be interpreted as "Emotional safety of interaction with children's".

The obtained data show that social workers, having sufficient professional competence and possessing the methods of emotional labor, can create conditions for the manifestation of the positive traits of children's. At the same time, interaction with them will be emotionally safe and comfortable for specialists.

And only in the third place (12\%) the factor represented by positive characteristics of the children's image is, and it can be interpreted as "Emotional acceptance of the children". This means that the child is emotionally perceived as a part of the specialist, close, pleasant.

Table 3 Factor "Emotional acceptance of the children"

\begin{tabular}{|l|c|}
\hline \multicolumn{1}{|c|}{ Statements } & Factor loads \\
\hline cruel - kind-hearted & $-0,59$ \\
\hline outsiders-insiders & $-0,58$ \\
\hline unpleasant-pleasant & $-0,57$ \\
\hline incomprehensible- comprehensible & $-0,51$ \\
\hline far-close & $-0,50$ \\
\hline unstable-stable & $-0,48$ \\
\hline evil-good & $-0,41$ \\
\hline unorganized-organized & $-0,38$ \\
\hline useless-useful & $-0,34$ \\
\hline irresponsible-responsible & $-0,23$ \\
\hline irritating-soothing & $-0,22$ \\
\hline
\end{tabular}

If the social worker sees these traits in the children, he/she feels sympathy for him/her and, accordingly, demonstrates a friendly attitude towards him/her. A friendly attitude towards a child is, first of all, respect for his/her personality, interest in his/her destiny, pedagogical optimism. It constantly manifests itself in the appeal to the children, in the process of using certain methods of pedagogical influence, such as request, encouragement, organization of success in learning, expectation of better results, persuasion, trust, etc. In this way the intensity of the emotional labor of the social worker is the smallest.

To correlate these problems with children's' perceptions, the correlation analysis was carried out using the rank correlation coefficient of Spearman, while a reliable critical value of 0.306 at a significance level of 0,005 was taken into account. 
The analysis of the correlation matrix showed that the emotionally negative perception of children's has a reliable connection with the allocation of discipline as the main problem ( $\mathrm{r}=0.521, \mathrm{p}=0.005)$. This situation can be based on a totalitarian understanding of the essence of pedagogical interaction: the child in the educational process is initially prescribed "what to be", he/she is not accepted by social workers as he/she is, for totalitarian consciousness openly declares contempt for any non normality.

It was also established the relationship between the identification of specialists the problems of the organization of the therapeutic environment (establishing contact between social assistance subjects, involving parents in the rehabilitation process, creating opportunities for self-realization for children's, etc.) and emotional acceptance of children's $(r=0.427, p=0.005)$. This dependence shows that if children's are emotionally perceived by social workers as close, pleasant, then there are no problems in interacting with them, establishing contact, organizing discipline $(r=0,371, p=0,005)$. Social workers with this kind of perception of children are able to see the essential aspects of social assistance.

High correlation values are also noted between the lack of identification of problems (in the interview a part of the respondents stated that they have no difficulties in their work) and fixation of perception in the sphere of disbelief in the ability of children's (superficial, not serious, unstable, etc.) $(r=0.354$, $\mathrm{p}=0.005$ ). In this case, one can conclude that a number of social workers do not believe in the success of social assistance to children, which results in the lack of desire to put their back into it and solve problems.

Also, emotional perception of the traits of children's, which relate to the possibility of emotionally safe interaction, and fixing problems in the field of negative traits in the children's (aggression, immorality, etc.) have high correlation values $(r=0.317, p=0.005)$. Such specialists, as a rule, have in their activities a shift in emphasis towards dealing with demerits of the child instead of encouraging merits. At the same time, the technology of social assistance to children sets the task not "to fight with the bad", but to find positive and develop it to such an extent that it will become an alternative to destructive behavior.

Conclusions about the dependence of the intensity of the emotional labor of the social worker on the pole of emotional perception of the client are confirmed by earlier studies. So Borgatta, Fanshel and Meyer (1960) proved that the perception of the client by the specialist directly affects the effectiveness of social work. Ashkanasy N. (2000) showed the role of emotions in professional activity.

N. Kader (2006) says that qualities such as empathy and sympathy for the client contribute to the full satisfaction of social workers with their professional activities, despite the high risk of stress and low material rewards. 
Modern researches also prove this fact. For example, in the study of Roh, Chul-Young, Moon, Yang, Seung-Bum \& Kwangho (2015), the determinants of the emotional labor of social workers in healthcare organizations were identified and it was concluded that managing emotions contributes to job satisfaction and burnout prevention of social workers.

O. Simonova (2017) proved that the analysis of emotional labor in the field of social assistance leads to a deeper and more complete understanding of its specifics and ethics of interaction with the client, helps to understand the importance of the moral mission of provision of social assistance to those in need.

The results of the study of T. Kanasz and I Zeilinska (2017) indicate that the skills of social workers used in managing emotions depend on three factors: the individual psychological characteristics of the social worker, the availability of institutionalized and informal support and professional experience. This conclusion does not contradict the results obtained by us, but we believe that the knowledge of the client's age and social and psychological characteristics, understanding the nature of the client's emotions, understanding the context of his/her life situation are no less important. This confirmed by our previous research (Tarkhanova \& Koryakovtseva, 2018).

The conclusion of the need to train social workers the psychological bases of working with clients is confirmed in E. Kume's study (2016), in which such training is called one of the twelve most important factors of social workers' satisfaction with their labor.

Thus, our conclusions do not contradict modern researches in the field of social work.

\section{Conclusions}

The conducted research has shown that the intensity of emotional labor of social workers providing social assistance to children depends on their emotional perception of children's. If children are initially perceived emotionally positively by the specialist, then there is no problem in interacting with them, establishing contact, organizing discipline. If children's are perceived emotionally negatively, then the social worker is forced to spend a lot of effort in regulating his/her emotions, experiencing emotional dissonance, his/her emotional labor becomes very intense, which can lead to low job satisfaction and emotional burnout in the future.

Based on the results of the study, we can conclude that to reduce the intensity of emotional labor of social workers who provide social assistance to children, it is necessary to increase their professional competence in the field of 
pedagogical communication, understanding the essence of age characteristics of behavior of children's, psychology of children in difficult life situations.

\section{Summary}

The relevance of the study is determined by the fact that social assistance to children potentially involved the danger of heavy experiences associated with dissatisfaction with professional activities and the probability of occupational stress. Refusal of expression in communication with children, the ability to perceive their behavior through the prism of social assistance, is a heavy emotional labor. Skills to understand own feelings and manage emotions today are considered by many researchers as professionally important qualities of the social worker. The purpose of this article is to study the emotional perception of children's by social workers and on the basis of the specifics of this perception to define the essence and content of emotional labor of social workers helping children.

The research is based on the concept of emotional labor of A.R. Hochschild, in which it is justified that the work on their emotions is an integral part of the professional activities of some professions, that is, it is, unfortunately, part of the labor that is lowly paid in Russia. Emotional labor is especially noticeable in those professions that are characterized by "face to face" interaction, the desire to influence their emotional state on the emotional state of clients.

A specific part of the emotional labor of the social worker who helps children is interaction with undisciplined children's. The intensity of emotional labor in this case depends on what the children are in the perception of the social worker. The identification of the subjective image of the child in need of social assistance was carried out by us using the scales of the semantic differential. The research was conducted in January-March 2018, 98 social workers working in the services to help children in a difficult life situation took part in it. Sex of respondents is: $93 \%$ of women, $7 \%$ of men; work experience in social services is from 3 to 18 years.

The conducted research has shown that the intensity of emotional labor of social workers providing social assistance to children depends on their emotional perception of children's. If children are initially perceived emotionally positively by the specialist, then there is no problem in interacting with them, establishing contact, organizing discipline. If children's are perceived emotionally negatively, then the social worker is forced to spend a lot of effort in regulating his/her emotions, experiencing emotional dissonance, his/her emotional labor becomes very intense, which can lead to low job satisfaction and emotional burnout in the future. Based on the results of the study, we can conclude that to reduce the intensity of emotional labor of social workers who provide social assistance to children, it is necessary to increase their professional competence in the field of pedagogical communication, understanding of the essence of age characteristics of behavior of children's, psychology of children in difficult life situations.

The findings do not contradict modern researches in the field of social work.

\section{References}

Ashkanasy, N. (2000). Emotions in the Workplace: Research, Theory, and Practice. Connecticut/London, Quorum Books.

Bolton, S.C. (2009). Getting to the Heart of Emotional Labor Process: A Reply to Brook. Work, Employment and Society, 23(3), 549-560. 
Borgatta, E.F., Fanshel, D., \& Meyer, H.J. (1960). Social Workers' Perceptions of Clients A Study of the Caseload of a Social Agency. Russell Sage Foundation. New York, 1960. - 103.

Brook, P. (2009). The Alienated Heart: Hochschild's "Emotional Labor" Thesis and the Capitalist Politics of Alienation. Class and Capital, 33, 7-31.

Brook, P. (2009). In Critical Defence of “Emotional Labor”: Refuting Bolton's Critique of Hochschild's Concept. Work, Employment and Society, 23(3), 531-548.

Gruzdev, M, Kasakova, E., Kuznetsova, I., \& Tarkhanova, I. (2018). University Graduates' Soft Skills: the Employers' Opinion. European Journal of Contemporary Education, 7(4), 690-698.

Hochschild, A. (2003). The Managed Heart: Commercialization of Human Feeling, Berkeley. Univ. of California Press.

Hochschild, A. (2011). Emotional Life on the Market Frontier. Annu. Rev. Sociol., Palo Alto: Annual reviews, 37, 21-33.

Hochschild, A.R. (1979). Emotion Work, Feeling Rules, and Social Structure. American Journal of Sociology, 85, 551-575.

Kader, N. (2006). Job satisfaction of mental health social workers. British Journal of Psychiatry, 189(1), 84-84.

Kanasz, T., \& Zeilinska, I. (2017). Emotional Labor of the Polish Social Workers: The Study in Sociology of Emotions. Polish sociological review, 3(199), 352-365.

Korczynski, M. (2009). The Mystery Customer: Continuing Absences in the Sociology of Service Work. Sociology, 43(5), 952-967.

Kume, E. (2016). Job Satisfaction of Social Service Workers in Penitentiary Institutions in Albania. European Journal of Multidisciplinary Studies Articles, vol., 78-85.

Lively, K. J. (2008) Emotional Segues and the Management of Emotion by Women and Men. Social Forces, Chapel Hill: Oxford Univ. Press, no 87, 911-936.

Roh, Chul-Young, Moon, M., Seung-Bum, Y., \& Kwangho, J. (2015). Linking Emotional Labor, Public Service Motivation, and Job Satisfaction: Social Workers in Health Care Settings. Social work in public health, 31, 1-15.

Simonova, O.A. (2017). Emotion management and the professional culture of administrative social workers in Russia. Common standards versus the moral mission of social care, 15(1), 129-142.

Tarkhanova, I.Y., \& Koryakovtseva, O.A. (2018). The development of universal competences of the adult population through education. The European Proceedings of Social \& Behavioural Sciences, Volume LI, 1989-1996.

Taylor, P., \& Wingfield, A.H. (2010). Are Some Emotions Marked "Whites only"? Racialized Feeling Rules in Professional Workplaces. Social Problems, 57, 251-268.

Vandysheva, L.V. (2014). Emotional work of volunteers: analysis of experience of realization. Fundamental research, 9-4, 838-842. 